\title{
Self-avoiding walks and the Fisher transformation
}

\author{
Geoffrey Grimmett Zhongyang Li* \\ Statistical Laboratory \\ Centre for Mathematical Sciences \\ Cambridge University \\ Wilberforce Road \\ Cambridge CB3 0WB, UK \\ \{g.r.grimmett, z.li\}@statslab.cam.ac.uk \\ http://www.statslab.cam.ac.uk/ grg/ \\ http://www.statslab.cam.ac.uk/ zl296/
}

Submitted: Aug 25, 2012; Accepted: Sep 18, 2013; Published: Sep 26, 2013

Mathematics Subject Classifications: 05C30, 82B20

\begin{abstract}
The Fisher transformation acts on cubic graphs by replacing each vertex by a triangle. We explore the action of the Fisher transformation on the set of selfavoiding walks of a cubic graph. Iteration of the transformation yields a sequence of graphs with common critical exponents, and with connective constants converging geometrically to the golden mean.

We consider the application of the Fisher transformation to one of the two classes of vertices of a bipartite cubic graph. The connective constant of the ensuing graph may be expressed in terms of that of the initial graph. When applied to the hexagonal lattice, this identifies a further lattice whose connective constant may be computed rigorously.
\end{abstract}

Keywords: self-avoiding walk, connective constant, cubic graph, Fisher transformation, quasi-transitive graph.

\section{Introduction}

A self-avoiding walk (abbreviated to SAW) on a graph $G$ is a path that visits no point more than once. SAWs were introduced in the chemical theory of polymerization (see

${ }^{*}$ This work was supported in part by the Engineering and Physical Sciences Research Council under grant $\mathrm{EP} / 103372 \mathrm{X} / 1$. 
Flory [5]), and their critical behaviour has been studied since by mathematicians and physicists (see, for example, the book [14] of Madras and Slade). The exponential rate of growth of the number of SAWs is given by the so-called connective constant $\mu=\mu(G)$ of the graph. Only few graphs of interest have connective constants that are known exactly.

We explore the action of the Fisher transformation on the set of SAWs of a cubic graph $G$. The transformation maps $G$ to a new graph $F(G)$. We have two sets of results. First, the connective constants of $G$ and $F(G)$ satisfy a simple functional relation, and in addition, three of the principal critical exponents are invariant under the transformation. Secondly, under repeated applications of the Fisher transformation, the graphs converge to a version of the Sierpinski gasket, and the connective constants converge geometrically to the golden mean. See Theorems 1 and 2 for formal statements of our results.
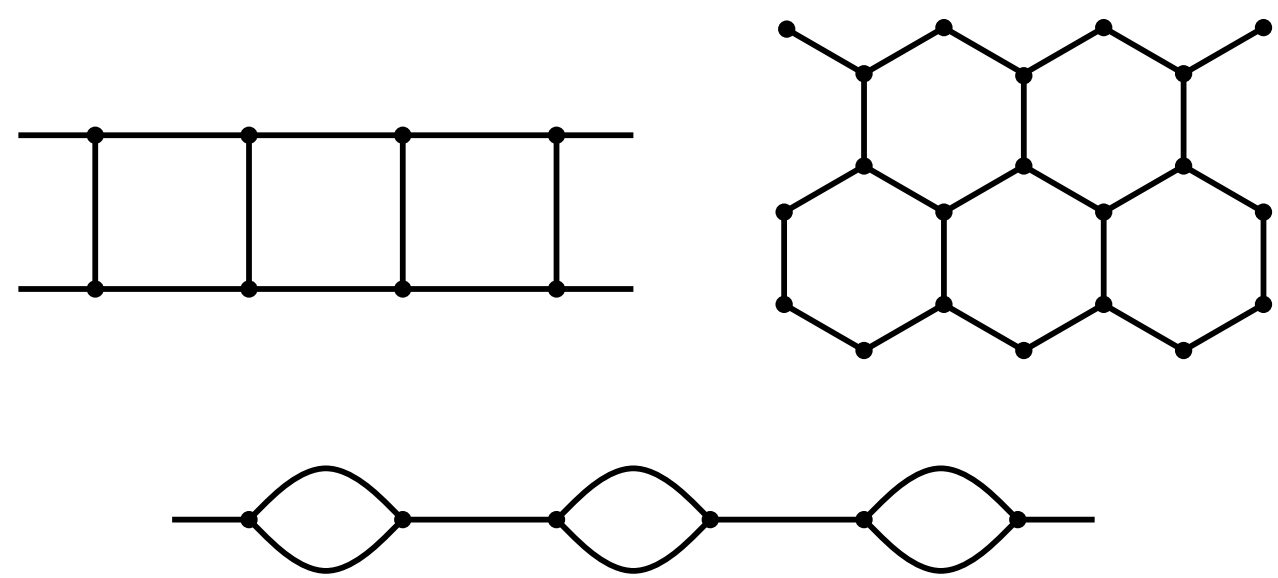

Figure 1: Three cubic graphs: the (doubly-infinite) ladder graph $\mathbb{L}$; the hexagonal lattice $\mathbb{H}$; the bridge graph $\mathbb{B}_{3}$ obtained from $\mathbb{Z}$ by joining every alternating pair of consecutive vertices by 2 parallel edges.

Our second set of results concerns the application of the Fisher transformation to a bipartite graph $G$ one of whose vertex-sets is cubic. As before, the ensuing connective constant may be expressed in terms of that of $G$, and the critical exponents are invariant. When applied to the hexagonal lattice $\mathbb{H}$ (see Figure 1), this yields the lattice $\widetilde{\mathbb{H}}$ illustrated in Figure 2. Nienhuis's proposed value $\mu(\mathbb{H})=\sqrt{2+\sqrt{2}}$ has been proved recently by Duminil-Copin and Smirnov [3], and the value of $\mu(\widetilde{\mathbb{H}})$ may be deduced rigorously from this, namely as the root of the equation

$$
x^{-3}+x^{-4}=\frac{1}{2+\sqrt{2}} .
$$

See Theorem 3.

Section 2 is devoted to basic definitions. The Fisher transformation, and its action on counts of SAWs, is described in Section 3, and our Theorems 1-3 are stated there. The proofs of results are found in Sections 4 and 5 . 


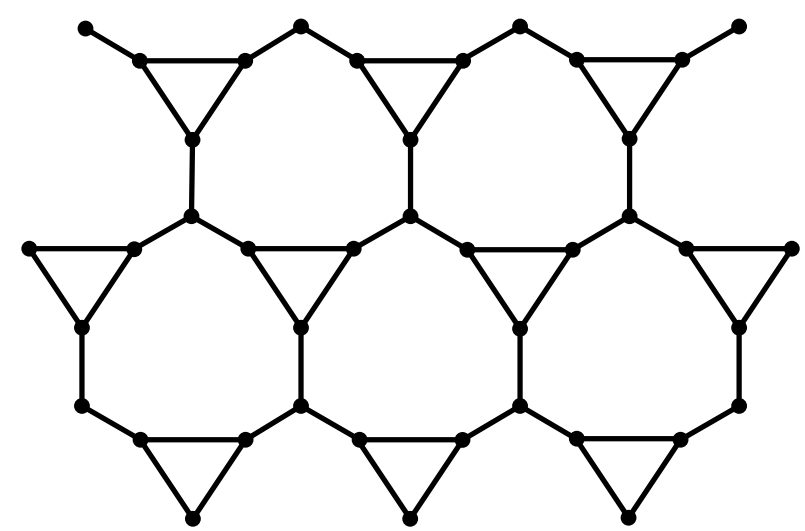

Figure 2: The lattice $\widetilde{\mathbb{H}}$ derived from the hexagonal lattice $\mathbb{H}$ by applying the Fisher transformation at alternate vertices. Its connective constant $\widetilde{\mu}$ is the root of the equation $x^{-3}+x^{-4}=1 /(2+\sqrt{2})$.

In the companion papers $[7,8,9]$, we study inequalities for the connective constants of regular graphs. For an infinite, connected, cubic, quasi-transitive graph $G$ (possibly with parallel edges), it is elementary that

$$
1 \leqslant \mu(G) \leqslant 2 .
$$

If such $G$ is vertex-transitive and simple (or non-simple and satisfying a certain condition), it is proved in $[7$, Thms $4.1,4.3]$ that $\sqrt{2} \leqslant \mu(G) \leqslant 2$, with equalities for the bridge graph $\mathbb{B}_{3}$ of Figure 1 and the 3 -regular tree, respectively.

\section{Notation}

All graphs studied henceforth in this paper will be assumed infinite, connected, and simple (in that they have neither loops nor multiple edges). An edge $e$ with endpoints $u, v$ is written $e=\langle u, v\rangle$. If $\langle u, v\rangle \in E$, we call $u$ and $v$ adjacent and write $u \sim v$. The degree of vertex $v$ is the number of edges incident to $v$, denoted $\operatorname{deg}(v)$. A graph is called cubic if all vertices have degree 3 . The graph-distance between two vertices $u, v$ is the number of edges in the shortest path from $u$ to $v$, denoted $d_{G}(u, v)$.

The automorphism group of the graph $G=(V, E)$ is denoted $\mathcal{A}=\mathcal{A}(G)$. The graph $G$ is called quasi-transitive if there exists a finite subset $W \subseteq V$ such that, for $v \in V$ there exists $\alpha \in \mathcal{A}$ such that $\alpha v \in W$. We call such $W$ a fundamental domain, and shall normally (but not invariably) take $W$ to be minimal with this property. The graph is called vertex-transitive (or transitive) if the singleton set $\{v\}$ is a fundamental domain for some (and hence all) $v \in V$.

A walk $w$ on $G$ is an alternating sequence $v_{0} e_{0} v_{1} e_{1} \cdots e_{n-1} v_{n}$ of vertices $v_{i}$ and edges $e_{i}$ such that $e_{i}=\left\langle v_{i}, v_{i+1}\right\rangle$. We write $|w|=n$ for the length of $w$, that is, the number of edges in $w$. 
Let $n \in \mathbb{N}$, the natural numbers. An $n$-step self-avoiding walk (SAW) on $G$ is a walk containing $n$ edges that includes no vertex more than once. Let $\sigma_{n}(v)$ be the number of $n$-step SAWs starting at $v \in V$. It was shown by Hammersley [10] that, if $G$ is quasitransitive, there exists a constant $\mu=\mu(G)$, called the connective constant of $G$, such that

$$
\mu=\lim _{n \rightarrow \infty} \sigma_{n}(v)^{1 / n}, \quad v \in V .
$$

It will be convenient to consider also SAWs starting at 'mid-edges'. We identify the edge $e$ with a point (also denoted $e$ ) placed at the middle of $e$, and then consider walks that start and end at these mid-edges. Such a walk is self-avoiding if it visits no vertex or mid-edge more than once, and its length is the number of vertices visited.

The minimum of two reals $x, y$ is denoted $x \wedge y$, and the maximum $x \vee y$.

\section{Fisher transformation}

Let $G=(V, E)$ be a simple graph and let $v \in V$ have degree 3. The so-called Fisher transformation acts on $v$ by replacing it by a triangle, as illustrated in Figure 3. This transformation has been valuable in the study of the relations between Ising, dimer, and general vertex models (see $[2,4,12,13]$ ), and more recently of SAWs on the Archimedean lattice denoted $\left(3,12^{2}\right)$ (see $[6,11]$ ). In the remainder of this paper, we make use of the Fisher transformation in the context of SAWs and the connective constant. It will be applied to cubic graphs, of which the hexagonal and square/octagon lattices are examples.
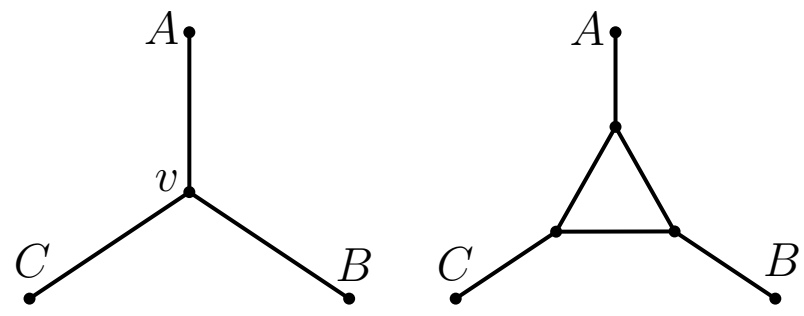

Figure 3: The Fisher triangulation of the star. Any triangle thus created is called a Fisher triangle.

It is convenient to work with graphs with well-defined connective constants, and to this end we assume that $G=(V, E)$ is quasi-transitive and connected, so that its connective constant is given by $(2)$. We write $F(G)$ for the graph obtained from the cubic graph $G$ by applying the Fisher transformation at every vertex. The automorphism group of $G$ induces an automorphism subgroup of $F(G)$. We write $\phi=\frac{1}{2}(\sqrt{5}+1)$ for the golden mean. The next theorem may be known to others.

Theorem 1. Let $G$ be an infinite, quasi-transitive, connected, cubic graph, and consider the sequence $\left(G_{k}: k=0,1,2, \ldots\right)$ defined by $G_{0}=G$ and $G_{k+1}=F\left(G_{k}\right)$. Then

(a) The connective constants $\mu_{k}$ of the $G_{k}$ satisfy $\mu_{k}^{-1}=g\left(\mu_{k+1}^{-1}\right)$ where $g(x)=x^{2}+x^{3}$. 
(b) The sequence $\mu_{k}$ converges monotonely to $\phi$, and

$$
-\left(\frac{4}{7}\right)^{k} \leqslant \mu_{k}^{-1}-\phi^{-1} \leqslant\left[\frac{1}{2}(7-\sqrt{5})\right]^{-k}, \quad k \geqslant 1 .
$$

Theorem 1 provokes the question of the existence of a graph limit of repeated application of the Fisher transformation. It is easily seen that the limiting graph comprises two copies of the Sierpinski gasket, as illustrated in Figure 4.

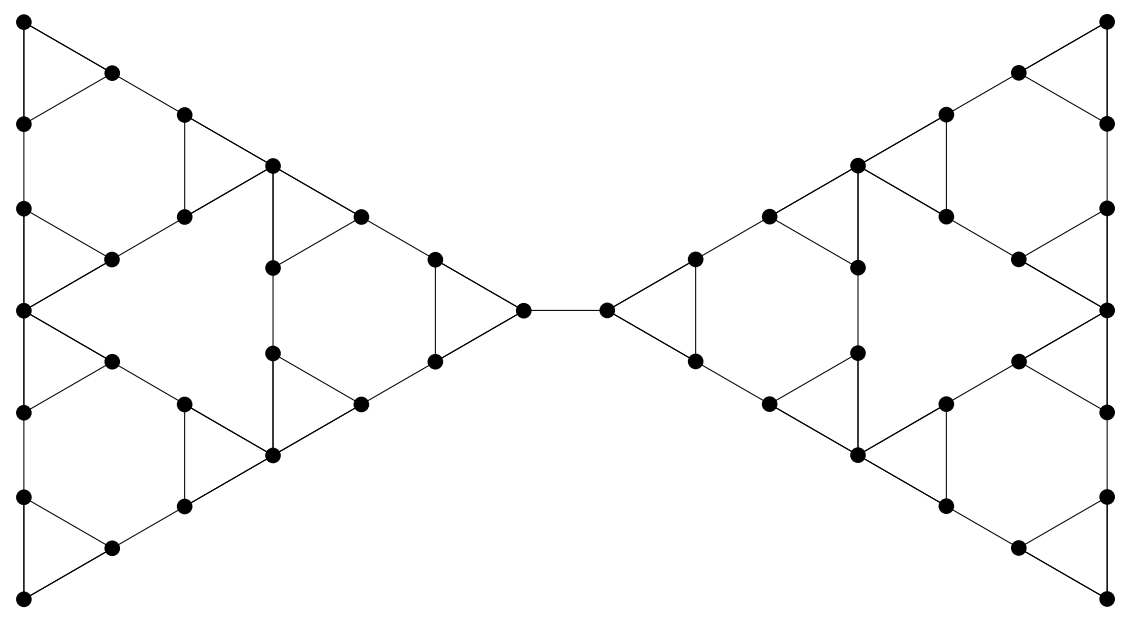

Figure 4: Through repeated application of the Fisher transformations to a single edge with endvertices of degree three, one arrives at a graph comprising two Sierpinski gaskets.

By Theorem 1(b), either $\mu_{k} \downarrow \phi$ or $\mu_{k} \uparrow \phi$. The decreasing limit holds if and only if $\mu_{0} \geqslant \phi$. We present no satisfactory characterization of graphs $G$ for which $\mu(G) \geqslant \phi$ beyond noting that this holds whenever $G$ contains as a subgraph a copy of a graph with connective constant $\phi$, such as the ladder graph $\mathbb{L}$ (or the semi-infinite ladder graph) of Figure 1. Furthermore, if $\mu(G)>\phi$ and $\widetilde{G}$ is obtained from $G$ by a sequence of Fisher transformations, then $\mu(\widetilde{G}) \geqslant \phi$. We ask whether $\mu(G) \geqslant \phi$ for any infinite, connected, vertex-transitive, simple, cubic graph $G$.

We turn to the topic of critical exponents, beginning with a general introduction for the case when there exists a periodic, locally finite embedding of $G$ into $\mathbb{R}^{d}$ with $d \geqslant 2$. The case of general $G$ has not not been studied extensively, and most attention has been paid to the hypercubic lattice $\mathbb{Z}^{d}$. It is believed (when $d \neq 4$ ) that there is a power-order correction, in the sense that there exists $A_{v}>0$ and an exponent $\gamma \in \mathbb{R}$ such that

$$
\sigma_{n}(v) \sim A_{v} n^{\gamma-1} \mu^{n} \quad \text { as } n \rightarrow \infty, \quad v \in V .
$$

Furthermore, the value of the exponent $\gamma$ is believed to depend on $d$ and not further on the choice of graph $G$. When $d=4$, (3) should hold with $\gamma=1$ and subject to the inclusion on the right side of the logarithmic correction factor $(\log n)^{1 / 4}$. See $[1,14]$ for accounts of critical exponents for SAWs. 
Let $v \in V$ and

$$
Z_{v, w}(x)=\sum_{n=0}^{\infty} \sigma_{n}(v, w) x^{n}, \quad w \in V, x>0,
$$

where $\sigma_{n}(v, w)$ is the number of $n$-step SAWs with endpoints $v, w$. It is known under certain circumstances that the generating functions $Z_{v, w}$ have radius of convergence $\mu^{-1}$ (see [14, Cor. 3.2.6]), and it is believed that there exists an exponent $\eta$ and constants $A_{v}^{\prime}>0$ such that

$$
Z_{v, w}\left(\mu^{-1}\right) \sim A_{v}^{\prime} d_{G}(v, w)^{-(d-2+\eta)} \quad \text { as } d_{G}(v, w) \rightarrow \infty .
$$

Let $\Sigma_{n}(v)$ be the set of $n$-step SAWs from $v$, and write $\langle\cdot\rangle_{n}^{v}$ for expectation with respect to uniform measure on $\Sigma_{n}(v)$. Let $\|\pi\|$ be the graph-distance between the endpoints of a SAW $\pi$. It is believed (when $d \neq 4$ ) that there exists an exponent $\nu$ and constants $A_{v}^{\prime \prime}>0$ such that

$$
\left\langle\|\pi\|^{2}\right\rangle_{n}^{v} \sim A_{v}^{\prime \prime} n^{2 \nu}, \quad v \in V
$$

As above, this should hold for $d=4$ with $\nu=\frac{1}{2}$ and subject to the inclusion of the correction factor $(\log n)^{1 / 4}$.

The above three exponents are believed to be related to one another through the so-called Fisher relation

$$
\gamma=\nu(2-\eta)
$$

The above discussion is not a suitable basis for a rigorous analysis of the critical exponents of general graphs. The first reason for this is that the exponents are introduced via hypotheses (3), (5), (6), and there is no proof of existence. The second is that (5) depends on the number $d$ of dimensions. It is convenient to work with definitions of critical exponents that do not depend on an assumption of dimensionality, and thus we proceed as follows.

Let $G$ be an infinite, connected, quasi-transitive graph with connective constant $\mu$ and fundamental domain $W$. Let $X$ be the set of edges incident to vertices in $W$, and let $\Sigma$ be the set of SAWs on $G$ starting at mid-edges in $X$. We define the function

$$
Y(x, y)=\sum_{\pi \in \Sigma} \frac{x^{|\pi|}}{|\pi|^{y}}, \quad x>0, y \in \mathbb{R},
$$

where the denominator is interpreted as 1 when $|\pi|=0$. For fixed $x, Y(x, y)$ is nonincreasing in $y$. Let $\gamma=\gamma(G) \in[-\infty, \infty]$ be such that

$$
Y\left(\mu^{-1}, y\right) \begin{cases}=\infty & \text { if } y<\gamma \\ <\infty & \text { if } y>\gamma\end{cases}
$$

We shall assume that $-\infty<\gamma<\infty$. It will be convenient at times to assume more about the number $\sigma_{n}$ of $n$-step SAWs from mid-edges in $X$, namely that there exist constants $C_{i}=C_{i}(W) \in(0, \infty)$ and a slowly varying function $L$ such that

$$
C_{1} L(n) n^{\gamma-1} \mu^{n} \leqslant \sigma_{n} \leqslant C_{2} L(n) n^{\gamma-1} \mu^{n}, \quad n \geqslant 1 .
$$


Let

$$
V(z)=\sum_{n=1}^{\infty} \frac{1}{n^{2 z+1}}\left\langle\|\pi\|^{2}\right\rangle_{n}, \quad z \in[-\infty, \infty],
$$

where $\langle\cdot\rangle_{n}$ denotes the uniform average over the set $\Sigma_{n}$ of $n$-step SAWs in $\Sigma$. Thus, $V(z)$ is non-increasing in $z$, and we let $\nu=\nu(G) \in[-\infty, \infty]$ be such that

$$
V(z) \begin{cases}=\infty & \text { if } z<\nu \\ <\infty & \text { if } z>\nu\end{cases}
$$

Let $\alpha W$ denote the image of $W$ under an automorphism $\alpha \in \mathcal{A}$, with incident edges $\alpha X$, and let

$$
Z_{\alpha}(x)=\sum_{\pi \in \Sigma(\alpha)} x^{|\pi|}
$$

where $\Sigma(\alpha)$ is the subset of $\Sigma$ containing SAWs ending at mid-edges in $\alpha X$. We hypothesize that there exists $\eta=\eta(G) \in[-\infty, \infty]$ such that, for any sequence of automorphisms $\alpha$ satisfying $d_{G}(W, \alpha W) \rightarrow \infty$

$$
Z_{\alpha}\left(\mu^{-1}\right) d_{G}(W, \alpha W)^{w} \begin{cases}\rightarrow 0 & \text { if } w<\eta \\ \rightarrow \infty & \text { if } w>\eta .\end{cases}
$$

The $\eta$ of (5) should agree with that defined here, subject to the addition of $d-2$.

It is easily seen that the values of $\gamma, \eta, \nu$ do not depend on the choice of fundamental domain $W$.

We consider now the effect on critical exponents of the Fisher transformation. Let $W_{0}$ be a minimal fundamental domain of $G_{0}:=G$, with incident edge-set $X_{0}:=X$ as above. Write $W_{1}=F\left(W_{0}\right)$, the set of vertices of the triangles formed by the Fisher transformation at vertices in $W_{0}$, and $X_{1}$ for the set of edges of $G_{1}$ incident to vertices in $W_{1}$. It may be seen that $W_{1}$ is a fundamental domain of $G_{1}$.

Theorem 2. Let $G_{0}$ be an infinite, quasi-transitive, connected, cubic graph. Assume that $\left|\gamma\left(G_{0}\right)\right|<\infty$ and that $\eta\left(G_{1}\right)$ exists.

(a) The exponents $\gamma, \eta$ of $G_{0}$ and $G_{1}$ are equal.

(b) Let $\sigma_{n, k}$ be the number of $n$-step $S A W s$ on $G_{k}$ from mid-edges in $X_{k}$. Assume the $\sigma_{n, k}$ satisfy (8) for constants $C_{i, k}$ and a common slowly varying function $L$. Then the exponents $\nu$ of $G_{0}$ and $G_{1}$ are equal.

Our final result concerns the effect of the Fisher transformation when applied to just one of the vertex-sets of a bipartite graph. Let $G=(V, E)$ be bipartite with vertex-sets $V_{1}, V_{2}$ coloured white and black, respectively. We think of $G$ as a graph together with a colouring $\chi$, and the coloured-automorphism group $\mathcal{A}_{\mathrm{c}}=\mathcal{A}_{\mathrm{c}}(G)$ of the pair $(G, \chi)$ is the set of maps $\phi: V \rightarrow V$ which preserve both graph structure and colouring. The coloured graph is quasi-transitive if there exists a finite subset $W \subseteq V$ such that: for all $v \in V$, there exists $\alpha \in \mathcal{A}_{\mathrm{c}}$ such that $\alpha v \in W$. As before, such a set $W$ is called a fundamental domain. 
Theorem 3. Let $G$ be an infinite, connected, bipartite graph with vertex-sets coloured black and white, and suppose that the coloured graph $G$ is quasi-transitive, and every black vertex has degree 3. Let $\widetilde{G}$ be obtained by applying the Fisher transformation at each black vertex.

(a) The connective constants $\mu$ and $\widetilde{\mu}$ of $G$ and $\widetilde{G}$, respectively, satisfy $\mu^{-2}=h\left(\widetilde{\mu}^{-1}\right)$ where $h(x)=x^{3}+x^{4}$.

(b) Under the corresponding assumptions of Theorem 2, the exponents $\gamma, \eta, \nu$ are the same for $G$ as for $\widetilde{G}$.

Theorem 3(a) implies an exact value of a connective constant that does not appear to have been noted previously. Take $G=\mathbb{H}$, the hexagonal lattice with connective constant

$\mu=\sqrt{2+\sqrt{2}} \approx 1.84776$, see [3]. The decorated lattice $\widetilde{\mathbb{H}}$ is illustrated in Figure 2, and has connective constant $\widetilde{\mu}$ satisfying $\mu^{-2}=h\left(\widetilde{\mu}^{-1}\right)$, which may be solved to obtain $\widetilde{\mu} \approx 1.75056$.

The proofs of Theorems 1-2 and 3 are found in Sections 4 and 5, respectively.

\section{Proof of Theorems 1-2}

Proof of Theorem 1. Let $G_{0}=\left(V_{0}, E_{0}\right)$ be an infinite, connected, quasi-transitive, cubic graph. The graph $G_{1}=F\left(G_{0}\right)$ is also quasi-transitive and cubic. It suffices for part (a) to show that the connective constants $\mu_{k}$ of the $G_{k}$ satisfy

$$
g\left(\mu_{1}^{-1}\right)=\mu_{0}^{-1} \text {. }
$$

By $(1), \mu_{k} \in[1,2]$ for $k=1,2$.

Let $W_{0}$ be a minimal fundamental domain of $G_{0}$, and let $X_{0}$ be the subset of $E_{0}$ comprising all edges incident to vertices in $W_{0}$. Write $W_{1}=F\left(W_{0}\right)$, the set of vertices of the triangles formed by the Fisher transformation at vertices in $W_{0}$, and $X_{1}$ for the set of edges of $G_{1}$ incident to vertices in $W_{1}$. It may be seen that $W_{1}$ is a fundamental domain of $G_{1}$.

It is convenient to work with SAWs that start and end at mid-edges. Note that the mid-edges of $E_{0}$ (respectively, $X_{0}$ ) may be viewed as mid-edges of $E_{1}$ (respectively, $X_{1}$ ).

For $k=0,1$, the partition functions of SAWs on $G_{k}$ are the polynomials

$$
Z_{k}(x)=\sum_{\pi \in \Sigma_{k}} x^{|\pi|}, \quad x>0
$$

where the sum is over the set $\Sigma_{k}$ of SAWs starting at mid-edges of $X_{k}$. Similarly, we set

$$
Z_{1}^{*}(x)=\sum_{\pi \in \Sigma_{1}^{*}} x^{|\pi|}
$$


where the sum is over the set $\Sigma_{1}^{*}$ of SAWs on $G_{1}$ starting at mid-edges of $X_{0}$ and ending at mid-edges of $E_{0}$. For $k=0,1$,

$$
Z_{k}(x) \begin{cases}<\infty & \text { if } x<\mu_{k}^{-1} \\ =\infty & \text { if } x>\mu_{k}^{-1}\end{cases}
$$

The following basic argument formalizes a method known already in the special case of the hexagonal lattice, see for example $[6,11]$. Since $\Sigma_{1}^{*} \subseteq \Sigma_{1}$, we have

$$
Z_{1}^{*}(x) \leqslant Z_{1}(x)
$$

Let $N(\pi)$ be the number of endpoints of a SAW $\pi \in \Sigma_{1}$ that are mid-edges of $E_{0}$. The set $\Sigma_{1}$ may be partitioned into three sets.

(a) If $N(\pi)=2$, then $\pi$ contributes to $Z_{1}^{*}$.

(b) $\pi$ may be a walk within a single Fisher triangle.

(c) If (b) does not hold and $N(\pi) \leqslant 1$, any endpoint not in $E_{0}$ may be moved by one, two, or three steps along $\pi$ to obtain a shorter SAW in $\Sigma_{1}^{*}$.

By considering the numbers of SAWs in each subcase of (c), we find that

$$
Z_{1}(x) \leqslant\left[1+2 x+2 x^{2}+2 x^{3}\right]^{2} Z_{1}^{*}(x)+6\left|W_{0}\right|\left(1+x+x^{2}\right),
$$

where the last term corresponds to case (b). By (13)-(14),

$$
Z_{1}(x)<\infty \quad \Leftrightarrow \quad Z_{1}^{*}(x)<\infty
$$

so that, by (12),

$$
Z_{1}^{*}(x) \begin{cases}<\infty & \text { if } x<\mu_{1}^{-1} \\ =\infty & \text { if } x>\mu_{1}^{-1}\end{cases}
$$

With a SAW in $\Sigma_{1}^{*}$ we associate a SAW in $\Sigma_{0}$ by shrinking each Fisher triangle to a vertex. Each $n$-step SAW in $\Sigma_{0}$ arises thus from $2^{n}$ SAWs in $\Sigma_{1}^{*}$, because each triangle may be circumnavigated in either of 2 directions. Therefore,

$$
Z_{0}\left(x^{2}(1+x)\right)=Z_{1}^{*}(x),
$$

and (11) follows by (12) and (15).

We turn to Theorem 1(b). By $(1), \mu_{0}^{-1} \in\left[\frac{1}{2}, 1\right]$. The function $g$ is a bijection from $\left[\frac{1}{2}, 1\right]$ to $\left[\frac{3}{8}, 2\right]$. Furthermore, $g$ is strictly convex on $\left[\frac{1}{2}, 1\right]$ with fixed point $\phi^{-1}$. By (11) applied iteratively, $\mu_{k}^{-1} \rightarrow \phi^{-1}$ as $k \rightarrow \infty$, and the limit is monotone. The bounds on $\mu_{k}^{-1}-\phi^{-1}$ follow from the facts that $g^{\prime}\left(\frac{1}{2}\right)=\frac{7}{4}$ and $g^{\prime}\left(\phi^{-1}\right)=\frac{1}{2}(7-\sqrt{5})$. 
Proof of Theorem 2. Let

$$
Y_{k}(x, y)=\sum_{\pi \in \Sigma_{k}} \frac{x^{|\pi|}}{|\pi|^{y}}, \quad Y_{k}^{*}(x, y)=\sum_{\pi \in \Sigma_{k}^{*}} \frac{x^{|\pi|}}{|\pi|^{y}}, \quad x>0, y \in \mathbb{R},
$$

where the denominator is interpreted as 1 when $|\pi|=0$. Since $\Sigma_{1}^{*} \subseteq \Sigma_{1}$,

$$
Y_{1}^{*}(x, y) \leqslant Y_{1}(x, y) .
$$

Since every SAW in $\Sigma_{1} \backslash \Sigma_{1}^{*}$ either is an extension of a SAW in $\Sigma_{1}^{*}$ at the starting point, or endpoint (or both), by at most 3 steps, or is a short walk in a single Fisher triangle,

$$
Y_{1}(x, y) \leqslant 7^{|y|}\left[1+2 x+2 x^{2}+2 x^{3}\right]^{2} Y_{1}^{*}(x, y)+6\left|W_{0}\right|\left(1+x+\frac{x^{2}}{2^{y}}\right) .
$$

Therefore,

$$
Y_{1}^{*}(x, y)<\infty \quad \Leftrightarrow \quad Y_{1}(x, y)<\infty .
$$

As in the previous proof, any $n$-step SAW in $\Sigma_{0}$ gives rise to $2^{n}$ SAWs in $\Sigma_{1}^{*}$, and conversely any SAW in $\Sigma_{1}^{*}$ gives rise to a SAW in $\Sigma_{0}$ by shrinking each triangle to a vertex. For $n \geqslant 1$, the contribution of an $n$-step SAW $\pi \in \Sigma_{0}$ to $Y_{0}(x, y)$ is $x^{n} / n^{y}$, and to $Y_{1}^{*}(x, y)$ is

$$
T_{n}:=\sum_{l=0}^{n}\left(\begin{array}{l}
n \\
l
\end{array}\right) \frac{x^{2 n+l}}{(2 n+l)^{y}}
$$

Since

$$
C \frac{\left[x^{2}(1+x)\right]^{n}}{n^{y}} \leqslant T_{n} \leqslant D \frac{\left[x^{2}(1+x)\right]^{n}}{n^{y}},
$$

where $C=2^{-y} \wedge 3^{-y}$ and $D=2^{-y} \vee 3^{-y}$, we have that

$$
C \widetilde{Y}_{0}\left(x^{2}(1+x), y\right) \leqslant \widetilde{Y}_{1}^{*}(x, y) \leqslant D \widetilde{Y}_{0}\left(x^{2}(1+x), y\right),
$$

where $\widetilde{S}$ denotes the summation $S$ without the $n=0$ term. Therefore,

$$
Y_{1}^{*}(x, y)<\infty \quad \Leftrightarrow \quad Y_{0}\left(x^{2}(1+x), y\right)<\infty .
$$

By (17) and Theorem 1(a), $\gamma\left(G_{1}\right)=\gamma\left(G_{0}\right)$.

Let $\|\pi\|_{k}$ be the graph-distance between the endpoints of the walk $\pi$ on $G_{k}$. Assume $|\gamma|=\left|\gamma\left(G_{0}\right)\right|<\infty$, and write

$$
V_{k}(z)=\sum_{n=1}^{\infty} \frac{1}{n^{2 z+1}}\left\langle\|\pi\|_{k}^{2}\right\rangle_{n, k}
$$

where $\langle\cdot\rangle_{n, k}$ denotes the uniform average over the set $\Sigma_{n, k}$ of $n$-step SAWs of $G_{k}$ starting at mid-edges of $X_{k}$. Similarly,

$$
V_{1}^{*}(z)=\sum_{n=1}^{\infty} \frac{1}{n^{2 z+1}} \frac{\sigma_{n, 1}^{*}}{\sigma_{n, 1}}\left\langle\|\pi\|_{1}^{2}\right\rangle_{n, 1}^{*},
$$


where $\sigma_{:}^{*}=\left|\Sigma_{:}\right|$, and $\langle\cdot\rangle_{n, 1}^{*}$ averages over the subset $\Sigma_{n, 1}^{*}$ of $\Sigma_{n, 1}$ containing $n$-step SAWs of $G_{1}$ that start in $X_{0}$ and end in $E_{0}$. We assume there exist constants $C_{i, k} \in(0, \infty)$ and a slowly varying function $L$ such that

$$
C_{1, k} L(n) n^{\gamma-1} \mu_{k}^{n} \leqslant \sigma_{n, k} \leqslant C_{2, k} L(n) n^{\gamma-1} \mu_{k}^{n}, \quad k=1,2 .
$$

We shall in fact use slightly less than this.

Similarly to the proof of (17), by (19)-(20), there exists $C_{1}<\infty$ such that

$$
V_{1}^{*}(z) \leqslant V_{1}(z) \leqslant C_{1} V_{1}^{*}(z)
$$

whence

$$
V_{1}(z)<\infty \quad \Leftrightarrow \quad V_{1}^{*}(z)<\infty .
$$

The contribution of $\pi \in \Sigma_{n, 0}$ to $V_{0}(z)$ is

$$
\frac{1}{\sigma_{n, 0} n^{2 z+1}}\|\pi\|_{0}^{2}
$$

As explained previously, $\pi$ gives rise to $2^{n}$ SAWs on $G_{1}$, making an aggregate contribution of

$$
\sum_{l=0}^{n}\left(\begin{array}{l}
n \\
l
\end{array}\right) \frac{1}{\sigma_{2 n+l, 1}(2 n+l)^{2 z+1}}\left(2\|\pi\|_{0}\right)^{2}
$$

to $V_{1}^{*}(z)$. By $(20)$, there exist constants $C_{i}>0$ such that

$$
\begin{aligned}
\frac{C_{2}}{n^{\gamma-1} L(n)} \sum_{l=0}^{n}\left(\begin{array}{l}
n \\
l
\end{array}\right)\left(\frac{1}{\mu_{1}}\right)^{2 n+l} & \leqslant \sum_{l=0}^{n}\left(\begin{array}{l}
n \\
l
\end{array}\right) \frac{1}{\sigma_{2 n+l, 1}} \\
& \leqslant \frac{C_{3}}{n^{\gamma-1} L(n)} \sum_{l=0}^{n}\left(\begin{array}{l}
n \\
l
\end{array}\right)\left(\frac{1}{\mu_{1}}\right)^{2 n+l} .
\end{aligned}
$$

By Theorem 1(a),

$$
\sum_{l=0}^{n}\left(\begin{array}{l}
n \\
l
\end{array}\right)\left(\frac{1}{\mu_{1}}\right)^{2 n+l}=\left(\frac{1}{\mu_{0}}\right)^{n}
$$

so that

$$
C_{4}\left(2^{-2 z} \wedge 3^{-2 z}\right) V_{0}(z) \leqslant V_{1}^{*}(z) \leqslant C_{5}\left(2^{-2 z} \vee 3^{-2 z}\right) V_{0}(z) .
$$

Therefore, for $|z|<\infty$,

$$
V_{1}^{*}(z)<\infty \quad \Leftrightarrow \quad V_{0}(z)<\infty
$$

By $(21), \nu\left(G_{0}\right)=\nu\left(G_{1}\right)$.

Any $\alpha \in \mathcal{A}\left(G_{0}\right)$ acts in a natural way on $G_{1}=F\left(G_{0}\right)$. For $k=0,1$ and $\alpha \in \mathcal{A}$, let

$$
Z_{\alpha, k}(x)=\sum_{\pi \in \Sigma_{k}(\alpha)} x^{|\pi|}, \quad Z_{\alpha, k}^{*}(x)=\sum_{\pi \in \Sigma_{k}^{*}(\alpha)} x^{|\pi|}, \quad x>0,
$$


where $\Sigma_{k}(\alpha)$ (respectively, $\left.\Sigma_{k}^{*}(\alpha)\right)$ is the set of SAWs of $G_{k}$ from mid-edges of $X_{k}$ (respectively, $X_{k-1}$ ) to mid-edges of $\alpha X_{k}$ (respectively, $\alpha X_{k-1}$ ). Assume $X_{0}$ and $\alpha X_{0}$ are disjoint. As before,

$$
Z_{\alpha, 1}^{*}(x)=Z_{\alpha, 0}\left(x^{2}(1+x)\right)
$$

and, as in (14),

$$
Z_{\alpha, 1}^{*}(x) \leqslant Z_{\alpha, 1}(x) \leqslant\left[1+2 x+2 x^{2}+2 x^{3}\right]^{2} Z_{\alpha, 1}^{*}(x) .
$$

By (22) and Theorem 1(a), for $w \in \mathbb{R}$,

$$
\lim _{d_{0}\left(W_{0}, \alpha W_{0}\right) \rightarrow \infty}\left[Z_{\alpha, 0}\left(\mu_{0}^{-1}\right) d_{0}\left(W_{0}, \alpha W_{0}\right)^{w}\right]=\infty
$$

if and only if

$$
\lim _{d_{1}\left(W_{1}, \alpha W_{1}\right) \rightarrow \infty}\left[Z_{\alpha, 1}\left(\mu_{1}^{-1}\right) d_{1}\left(W_{1}, \alpha W_{1}\right)^{w}\right]=\infty,
$$

where $d_{k}=d_{G_{k}}$. It follows that $\eta\left(G_{0}\right)=\eta\left(G_{1}\right)$.

\section{$5 \quad$ Proof of Theorem 3}

Let $G=(V, E)$ be a coloured bipartite graph satisfying the given assumptions. The vertices of any SAW on $G$ are alternately black and white. The decorated graph $\widetilde{G}=$ $(\widetilde{V}, \widetilde{E})$ is obtained from $G$ by replacing each black vertex by a triangle, as illustrated in Figure 3. The set $\widetilde{V}$ is coloured in the natural way: white vertices remain white, and vertices of Fisher triangles are coloured black.

Let $W$ be a minimal fundamental domain of $G$, and let $X$ be the subset of $E$ comprising all edges incident to vertices in $W$. Write $\widetilde{W}=F(W)$, the set of vertices of the triangles formed by the Fisher transformations at black vertices in $W$, and $\widetilde{X}$ for the set of edges of $\widetilde{G}$ incident to vertices in $\widetilde{W}$. It may be seen that $\widetilde{W}$ is a fundamental domain for the coloured graph $\widetilde{G}$. Recall that the mid-edges of $E$ may be viewed as a subset of mid-edges of $\widetilde{E}$, and thus $E$ may be viewed as a subset of $\widetilde{E}$.

Let $s_{n}$ be the number of $n$-step SAWs of $\widetilde{G}$ starting at mid-edges in $\widetilde{X}$, and let $c_{n}$ be the number of $n$-step SAWs of $\widetilde{G}$ starting at a mid-edge of $X$ and ending at a mid-edge of $E$. It is immediate that

$$
c_{n} \leqslant s_{n} .
$$

Any SAW counted in $s_{n}$ either lies within a single Fisher triangle, or may be obtained by a $k$-step extension (with some $k \leqslant 3$ ) at one or both endpoints of some SAW counted in one of $c_{n}, c_{n-1}, c_{n-2}, c_{n-3}$. Therefore,

$$
s_{n} \leqslant c_{n}+4 c_{n-1}+8 c_{n-2}+12 c_{n-3}+18|W| .
$$

By (2), the limits $\lim _{n \rightarrow \infty} s_{n}^{1 / n}$ and $\lim _{n \rightarrow \infty} c_{n}^{1 / n}$ exist and, by (24)-(25), these limits are equal. 
A SAW is called even if it has even length. Let $\mathcal{E}$ be the set of SAWs on $G$ starting at mid-edges of $X$, and let $\mathcal{E}_{\mathrm{e}}$ be the subset of $\mathcal{E}$ comprising the even SAWs. Let $x, y>0$. Each step of a SAW on $G$ is assigned weight $x$ at a black vertex, and weight $y$ at a white vertex. Let

$$
Z(x, y)=\sum_{\pi \in \mathcal{E}} x^{\left|\pi_{\mathrm{b}}\right|} y^{\left|\pi_{\mathrm{w}}\right|}
$$

where $\left|\pi_{\mathrm{b}}\right|$ and $\left|\pi_{\mathrm{w}}\right|$ are the numbers of black and white vertices visited by $\pi$. Similarly, let

$$
Z_{\mathrm{e}}(x, y)=\sum_{\pi \in \mathcal{E}_{\mathrm{e}}}(x y)^{|\pi| / 2} .
$$

It is clear by a decomposition of paths that

$$
\begin{aligned}
Z_{\mathrm{e}}(x, y) & \leqslant Z(x, y) \\
Z(x, y)-Z_{\mathrm{e}}(x, y) & \leqslant(2 x+2 y)\left(1+Z_{\mathrm{e}}(x, y)\right) .
\end{aligned}
$$

Hence,

$$
Z_{\mathrm{e}}(x, y)<\infty \quad \Leftrightarrow \quad Z(x, y)<\infty .
$$

We now introduce a third partition function $\widetilde{Z}$, namely of the set $\widetilde{\mathcal{E}}$ of SAWs on $\widetilde{G}$ starting at the mid-edges of $X$ and ending at mid-edges of $E$. Each step of such a SAW traverses two half-edges, and is allocated a weight which depends on these half-edges. Let $p, q, r>0$. Whenever both half-edges belong to $\widetilde{E} \backslash E$, the weight is $p$; if one half-edge is in $E$ and the other in $\widetilde{E} \backslash E$, the weight is $q$; if both half-edges are in $E$, the weight is $r$. Then

$$
\widetilde{Z}(p, q, r):=\sum_{\pi \in \widetilde{\mathcal{E}}} p^{\left|\pi_{p}\right|} q^{\left|\pi_{q}\right|} r^{\left|\pi_{r}\right|},
$$

where $\left|\pi_{p}\right|$ is the number of $p$-steps, etc. By counting edges of the different types,

$$
\widetilde{Z}(p, q, r)=Z\left(q^{2}(1+p), r\right) .
$$

By (27),

$$
\widetilde{Z}(p, q, r)<\infty \quad \Leftrightarrow \quad Z_{\mathrm{e}}\left(q^{2}(1+p), r\right)<\infty
$$

By (26),

$$
Z_{\mathrm{e}}\left(q^{2}(1+p), r\right) \begin{cases}<\infty & \text { if } q^{2}(1+p) r<\mu^{-2} \\ =\infty & \text { if } q^{2}(1+p) r>\mu^{-2}\end{cases}
$$

whence the radius of convergence of $\widetilde{Z}(x, x, x)=\sum_{n \geqslant 0} c_{n} x^{n}$ is the root of the equation

$$
x^{3}(1+x)=\frac{1}{\mu^{2}} .
$$

Theorem 3(a) follows. Part (b) is proved in a similar manner to the proof of Theorem 2. 


\section{References}

[1] R. Bauerschmidt, H. Duminil-Copin, J. Goodman, and G. Slade, Lectures on selfavoiding-walks, Probability and Statistical Physics in Two and More Dimensions (D. Ellwood, C. M. Newman, V. Sidoravicius, and W. Werner, eds.), Clay Mathematics Institute Proceedings, vol. 15, CMI/AMS publication, 2012, pp. 395-476.

[2] C. Boutillier and B. de Tilière, The critical Z-invariant Ising model via dimers: locality property, Commun. Math. Phys. 301 (2011), 473-516.

[3] H. Duminil-Copin and S. Smirnov, The connective constant of the honeycomb lattice equals $\sqrt{2+\sqrt{2}}$, Ann. Math. 175 (2012), 1653-1665.

[4] M. E. Fisher, On the dimer solution of planar Ising models, J. Math. Phys. 7 (1966), 1776-1781.

[5] P. Flory, Principles of Polymer Chemistry, Cornell University Press, 1953.

[6] G. R. Grimmett, Three theorems in discrete random geometry, Probab. Surv. 8 (2011), 403-441.

[7] G. R. Grimmett and Z. Li, Bounds on the connective constants of regular graphs, Combinatorica (2013), arXiv:1210.6277.

[8] _ Counting self-avoiding walks, Proc. Tsinghua Sanya International Mathematics Forum, 2013, arXiv:1304.7216.

[9] _ Strict inequalities for connective constants of transitive graphs, (2013), arXiv: 1301.3091.

[10] J. M. Hammersley, Percolation processes II. The connective constant, Proc. Camb. Phil. Soc. 53 (1957), 642-645.

[11] I. Jensen and A. J. Guttman, Self-avoiding walks, neighbour-avoiding walks and trails on semiregular lattices, J. Phys. A: Math. Gen. 31 (1998), 8137-8145.

[12] Z. Li, Local statistics of realizable vertex models, Commun. Math. Phys. 304 (2011), 723-763.

[13] _ Critical temperature of periodic Ising models, Commun. Math. Phys. 315 (2012), 337-381.

[14] N. Madras and G. Slade, Self-Avoiding Walks, Birkhäuser, Boston, 1993. 